\title{
Growth models on the Bethe lattice
}

\author{
Abbas Ali Saberi* \\ Department of Physics, University of Tehran, Post Office Box 14395-547, Tehran, Iran \\ Institut für Theoretische Physik, Universität zu Köln, Zülpicher Str. 77, 50937 Köln, Germany \\ Institute for Research in Fundamental Sciences (IPM), \\ School of Particles and Accelerators, Post Office Box 19395-5531, Tehran, Iran
}

(Dated: August 18, 2018)

\begin{abstract}
I report on an extensive numerical investigation of various discrete growth models describing equilibrium and nonequilibrium interfaces on a substrate of a finite Bethe lattice. An unusual logarithmic scaling behavior is observed for the nonequilibrium models describing the scaling structure of the infinite dimensional limit of the models in the Kardar-Parisi-Zhang (KPZ) class. This gives rise to the classification of different growing processes on the Bethe lattice in terms of logarithmic scaling exponents which depend on both the model and the coordination number of the underlying lattice. The equilibrium growth model also exhibits a logarithmic temporal scaling but with an ordinary power law scaling behavior with respect to the appropriately defined lattice size. The results may imply that no finite upper critical dimension exists for the KPZ equation.

PACS numbers: 05.40.-a, 68.35.Rh
\end{abstract}

The Kardar-Parisi-Zhang (KPZ) equation [1] is a simple nonlinear Langevin equation that describes the macroscopic properties of a wide variety of nonequilibrium growth processes $[2,3]$. This equation is also related to many other important physical problems such as the Burgers equation [4], dissipative transport in the driven-diffusion equation [5] and directed polymers in a random medium [6-8]. The KPZ equation for a stochastically growing interface described by a single valued height function $h(\mathbf{x}, t)$ on a $d$-dimensional substrate $\mathbf{x}$, is

$$
\partial_{t} h(\mathbf{x}, t)=\nu \nabla^{2} h+\frac{\lambda}{2}(\nabla h)^{2}+\eta(\mathbf{x}, t),
$$

where the first term represents relaxation of the interface caused by a surface tension $\nu$, the second describes the nonlinear growth locally normal to the surface, and the last is an uncorrelated Gaussian white noise in both space and time with zero average $\langle\eta(\mathbf{x}, t)\rangle=0$ and $\left\langle\eta(\mathbf{x}, t) \eta\left(\mathbf{x}^{\prime}, t^{\prime}\right)\right\rangle=2 D \delta^{d}\left(\mathbf{x}-\mathbf{x}^{\prime}\right) \delta\left(t-t^{\prime}\right)$, mimicking the stochastic nature of the growth process. The steady state interface profile is usually described in terms of the roughness: $w=\sqrt{\left\langle h^{2}(\mathbf{x}, t)\right\rangle-\langle h(\mathbf{x}, t)\rangle^{2}}$ which for a system of size $L$ behaves like $L^{\alpha} f\left(t / L^{\alpha / \beta}\right)$, where $f(x) \rightarrow$ const as $x \rightarrow \infty$ and $f(x) \sim x^{\beta}$ as $x \rightarrow 0$, so that $w$ grows with time like $t^{\beta}$ until it saturates to $L^{\alpha}$ when $t \sim L^{\alpha / \beta}$. $\alpha$ and $\beta$ are the roughness and the growth exponents, respectively, whose exact values are known only for the special case $d=1$ as $\alpha=1 / 2$ and $\beta=1 / 3$. The ratio $\bar{z}=\alpha / \beta$ is called dynamic exponent. A scaling relation $\alpha+\bar{z}=2$ follows from the invariance of Eq. (1) to an infinitesimal tilting of the surface which retains only one independent exponent, say $\alpha$, in the KPZ dynamics.

It is well known that for dimensions $d \leq 2$ the surface is always rough, while for $d>2$, the equation (1)

*Electronic address: ab.saberi@ut.ac.ir shows two different regimes in terms of the dimensionless strength of the nonlinearity coefficient whose critical value $\lambda_{c}$ separates flat and rough surface phases. In the weak coupling (flat) regime $\left(\lambda<\lambda_{c}\right)$ the nonlinear term is irrelevant and the behavior is governed by the $\lambda=0$ fixed point i.e., the linear Edward-Wilkinson (EW) equation [9], for which the exponents are known exactly: $\alpha=(2-d) / 2$ and $\beta=(2-d) / 4$. In the more challenging strong-coupling (rough) regime $\left(\lambda>\lambda_{c}\right)$, where the nonlinear term is relevant, the behavior of the KPZ equation is quite controversial and characterized by anomalous exponents. There is, however, a longstanding controversy concerning the existence and the value of an upper critical dimension $d_{c}$ above which, regardless of the strength of the nonlinearity, the surface remains flat.

At odds with many theoretical discussions supporting the existence of a finite upper critical dimension [10] between three and four [11, 12], and an analytical evidence that $d_{c}$ is bounded from above by four [13], or many others suggesting $d_{c} \approx 2.5$ [14] or $d_{c}=4[15,16]$, there is nevertheless a long list of evidence questioning these suggestions [17-23], some of which concluded that no finite upper critical dimension exists at all (for the most recent study, see [23]).

Here I study the infinite dimensional properties of growth models from two different KPZ and EW classes and compare them to realize whether the nonlinear term in (1) is relevant in this limit. It is inspired by the fact that if the nonlinear term is irrelevant in infinite dimensions, then one would expect the same statistical behavior for the models coming from each of the two classes. The result would shed a light on the existence of the upper critical dimension for the KPZ equation. To this aim, I investigate two discrete nonequilibrium models, ballistic deposition (BD) and restricted solid-on-solid (RSOS) models which are believed to be in the KPZ class [2, 2428], as well as an equilibrium model, random deposition with surface relaxation (RDSR) [29], which belongs to 


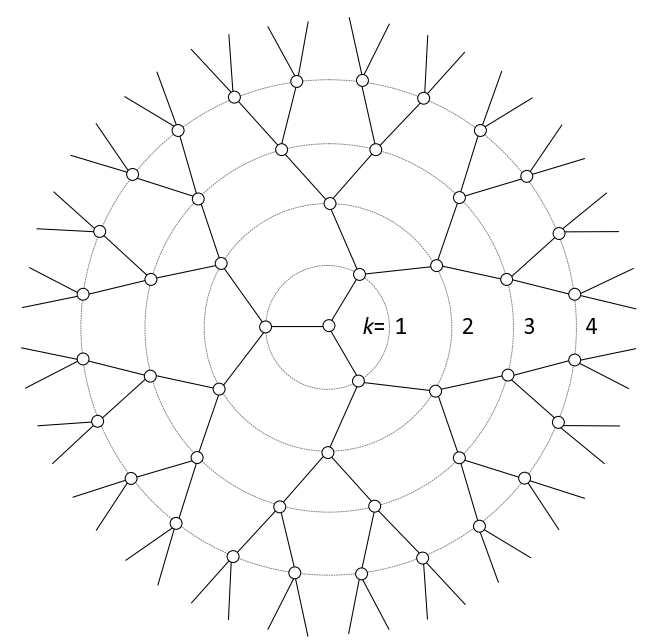

FIG. 1: Part of a Bethe lattice with coordination number $z=3$ embedded in the plane which is considered here as a substrate of different growth models. The vertically incident particles can land at the top of the lattice sites represented by open small circles at different shells $k=0,1,2, \cdots$. For a given finite lattice of fixed size $k$, one lattice site is randomly chosen at each step and a particle is added to that site which can either increase the height according to the standard rules of BD and RSOS models, or it can diffuse through the neighboring edges until it finds the column with a local minima in the searched area according to the RDSR model.

the EW class, all defined on the Bethe lattice, an effectively infinite dimensional lattice. I find that the models from different universality classes correspond to different statistical growth properties and scaling behavior, the evidence that questions the existence of a finite upper critical dimension for the KPZ equation.

Due to its distinctive topological structure, several statistical models involving interactions defined on the Bethe lattice [30] are exactly solvable and computationally inexpensive [31]. Various systems including magnetic models [30], percolation [32-35], nonlinear conduction [36], localization [32, 37], random aggregates [38, 39] and diffusion processes [40-42] have been studied on the Bethe lattice whose analytic results gave important physical insights to subsequent developments of the corresponding research fields. The Bethe lattice is defined as a graph of infinite points each connected to $z$ neighbors (the coordination number) such that no closed loops exist in the geometry (see Fig. 1). A finite type of the graph with boundary is also known as a Cayley tree and possesses the features of both one and infinite dimensions: since $N_{k}$, the total number of sites in a Bethe lattice with $k$ shells, is given as $N_{k}=\left[z(z-1)^{k}-2\right] /(z-2)$, the lattice dimension defined by $d=\lim _{k \rightarrow \infty}\left[\ln N_{k} / \ln k\right]$ is infinite. It is therefore often mentioned in the literature that the Bethe lattice describes the infinite-dimensional limit of a hypercubic lattice. As the lattice grows the number of sites in the surface, or the last shell, grows exponen- tially $z(z-1)^{k-1}$. Therefore, as the number of shells tends to infinity, the proportion of surface sites tends to $(z-2) /(z-1)$. By surface boundary we mean the set of sites of coordination number unity, the interior sites all have a coordination number $z$. Thus the vertices of a Bethe lattice can be grouped into shells as functions of the distances $k$ from the central vertex. Here $k$ is the number of bonds of a path between the shell and the central site and will be used as a measure of lattice size.

I have carried out extensive simulations of the $\mathrm{BD}$, RSOS and RDSR models on a finite Bethe lattice of different size $k$ and different coordination number $z$ (Fig. $1)$. I will first compute the surface width $w(t, k)$ as a function of time $t$ and examine its various scaling properties. For a given lattice size $k$, each Monte Carlo time step is defined as the time required for $N_{k}$ particles to deposit on the surface. I show that the surface widths for the models feature a normal behavior as for a typical growth model on a regular lattice: $w$ increases fast and finally saturates to a fixed value $w_{s}$. Nevertheless, the best fit to our data at early time before saturation shows that $w$ does not increase algebraically with time $\left(w \sim t^{\beta}\right)$, as is usually observed for growth models on ordinary lattices. Rather I find a logarithmic scaling behavior $w \sim \ln (t)^{\beta}[45]$ for all considered models. I also find that the saturated width $w_{s}$ of the interface for two $\mathrm{BD}$ and RSOS models behaves like a logarithmic scaling law $w_{s} \sim \ln (k)^{\alpha}$, while for the equilibrium RDSR model, it shows an ordinary power law behavior with the lattice

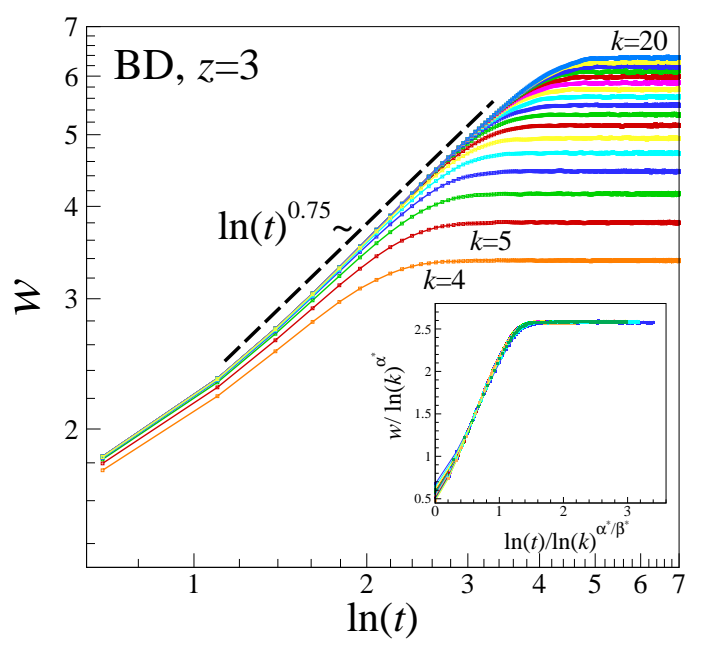

FIG. 2: (Color online) Surface width $w(t, k)$ for the BD model on a finite Bethe lattice of coordination number $z=3$, as a function of logarithm of time, for the seventeen different sizes, from the $k=4$ th to the 20th generation. Inset: Data collapse for the same data with $k>6$. The time is rescaled by $\ln (k)^{z^{*}}$ (having assumed $\bar{z}^{*}=\alpha^{*} / \beta^{*}=0.825 / 0.75=1.1$ ), and the width is rescaled by $\ln (k)^{\alpha^{*}}$ with $\alpha^{*}=0.825$. 


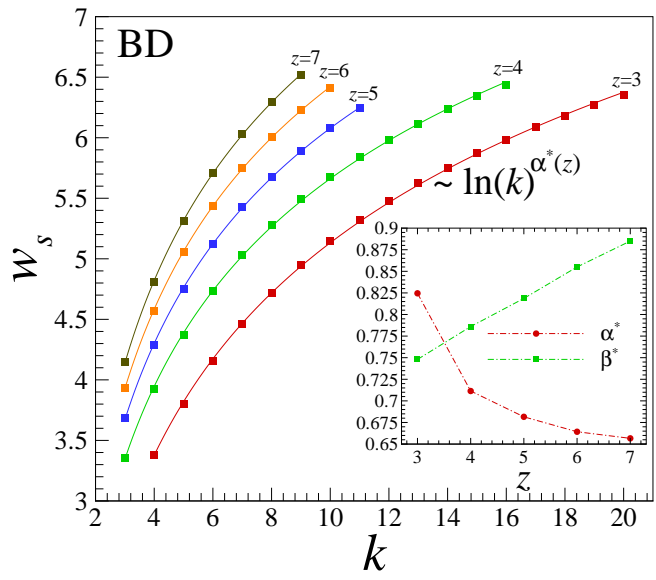

FIG. 3: (Color online) Main: saturated surface widths as functions of lattice size $k$, for the $\mathrm{BD}$ model on the finite Bethe lattices of different coordination number $z=3,4,5$, 6 and 7. The solid lines show the best logarithmic fits of form $w_{s} \sim \ln (k)^{\alpha^{*}(z)}$ to the data. Inset: the growth $\alpha^{*}$ and roughness $\beta^{*}$ exponents as functions of $z$.

size, $w_{s} \sim k^{\alpha}$. The model-dependent exponents are also found to be functions of the coordination number of the underlying lattice, $\alpha^{i}(z)$ and $\beta^{i}(z)$, where $\mathrm{i}=*$, $\star$ and $\circ$ denotes for BD, RSOS and RDSR models, respectively. Let me call $\alpha^{i}(z)$ and $\beta^{i}(z)$, roughness and growth exponents, respectively. This different scaling form with respect to the finite-dimensional case can be associated to the exponential (instead of polynomial) growth of the volume of a shell as a function of its radius on the Bethe lattice.

I first consider the BD model on a lattice with $z=3$. Fig. 2 shows the surface width $w(t, k)$ as a function of logarithm of time, for the seventeen different sizes, from the 4 th to the 20th generation. At early times before saturation, the data falls onto a straight line in a log-log scale indicating that the surface width initially increases algebraically with the logarithm of time as $w \sim \ln (t)^{\beta^{*}}$, with $\beta^{*}(z=3) \simeq 0.75(2)$. The best fit to the saturated width $w_{s}$ as a function of different lattice size, gives a scaling relation $w_{s} \sim \ln (k)^{\alpha^{*}}$, with $\alpha^{*}(z=3) \simeq 0.825(10)$.

As shown in the inset of Fig. 2, by standard rescaling of the parameteres, all curves collapse onto a single function.

In order to see how these exponents depend on $z$, series of extensive simulations were performed for the $\mathrm{BD}$ model on a Bethe lattice with different coordination number $z=3,4,5,6$ and 7 . For each $z$, the surface width $w(t, k)$ was measured for different lattice size $k$. Fig. 3 illustrates the saturated surface widths as functions of the lattice size for each coordination number. The solid lines in the figure show the best logarithmic fits of form $w_{s} \sim \ln (k)^{\alpha^{*}(z)}$ to the data, assigning a $z$-dependent roughness exponent $\alpha^{*}$ to each data set. I also find that the growth exponent $\beta^{*}$ is dependent on the coordination number of the substrate lattice. $\alpha^{*}(z)$ and $\beta^{*}(z)$ are plotted in the inset of Fig. 3. As can be seen, $\alpha^{*}$ decreases, while $\beta^{*}$ increases almost linearly with $z$.

To see whether such a logarithmic scaling behavior is a characteristic feature of the nonequilibrium growth models on the Bethe lattice, I have also measured the surface width for the RSOS model, for the seventeen different sizes, from the $k=4$ th to the 20th generation, and for $z=3$. The results are shown in Fig. 4. These suggest the same scaling behavior but with different estimated roughness and growth exponents $\alpha^{\star}(z=3) \simeq 0.90(1)$ and $\beta^{\star}(z=3) \simeq 0.57(2)$, respectively. The exponents for this model depend again on the coordination number but both are decreasing with $z$. For $z=4$, the exponents are estimated as $\alpha^{\star}(z=4) \simeq 0.68(1)$ and $\beta^{\star}(z=4) \simeq 0.47(2)$ (see inset of Fig. 4 ).

In random deposition with surface relaxation (RDSR) $[29,43]$, each particle is randomly dropped onto the surface, and it is allowed to diffuse around on the surface within a prescribed region about the deposited column, until it finds the column with a local minima in the searched area. The corresponding continuum model is the EW equation [9].

Fig. 5 summarizes the results obtained from implementing the RDSR model on a finite Bethe lattice of different size from the $k=4$ th to the 16 th generation with $z=3$. The first remarkable observation is that the crossover time to the steady state is quite larger than that needed

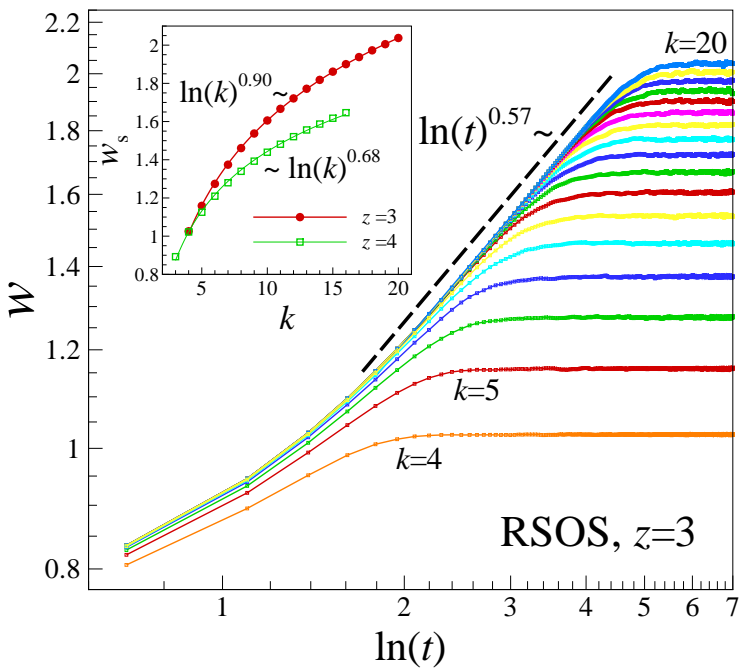

FIG. 4: (Color online) Main: surface width $w(t, k)$ for the RSOS model on a finite Bethe lattice of coordination number $z=3$, as a function of logarithm of time, for the seventeen different sizes, from the $k=4$ th to the 20 th generation. Inset: saturated surface widths as functions of lattice size $k$, for two different coordination numbers $z=3$ and 4 . 


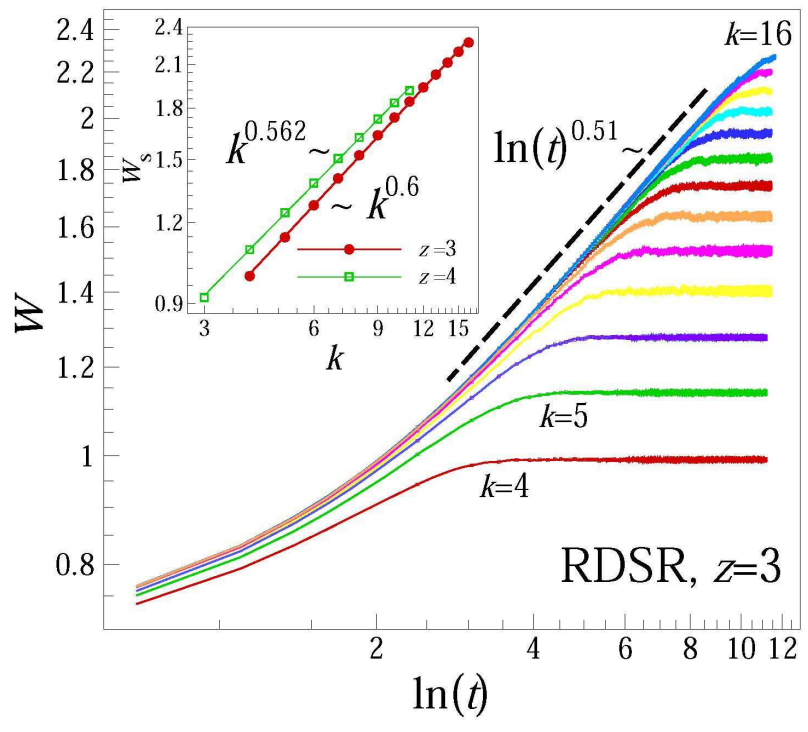

FIG. 5: (Color online) Main: surface width $w(t, k)$ for the equilibrium RDSR model on a finite Bethe lattice of coordination number $z=3$, as a function of logarithm of time, for the thirteen different sizes, from the $k=4$ th to the 16 th generation. Inset: saturated surface widths as functions of lattice size $k$, for two different coordination numbers $z=3$ and 4. Unlike the nonequilibrium BD and RSOS models, $w_{s}$ here shows a power law scaling behavior $w_{s} \sim k^{\alpha^{\circ}(z)}$, with the lattice size $k$.

for the above discussed nonequilibrium models, growing exponentially with $k$ in this case. Therefore, the CPU time required for simulations to reach the desired accuracy is orders of magnitude higher. As shown in Fig. 5, the temporal logarithmic scaling behavior for this model again holds. I find the scaling relation $w \sim \ln (t)^{\beta^{\circ}(z)}$, with $\beta^{\circ}(z=3) \simeq 0.51(2)$.

The most remarkable scaling feature observed in the RDSR model is that, unlike the two BD and RSOS models, the average saturated width $w_{s}$ has no longer a power law relation with the logarithm of the size, but with the size $k$ itself. The resulting data is plotted in the inset of Fig. 5. I find that $w_{s} \sim k^{\alpha^{\circ}(z)}$, with $\alpha^{\circ}(z=3) \simeq 0.60(1)$. The roughness and growth exponents again depend on the coordination number. Simulations for the RDSR model on a finite Bethe lattice of different size from $k=3$ th to the 14th generation for $z=4$, provide a satisfactory estimation of the exponents: $\alpha^{\circ}(z=4) \simeq 0.562(10)$ (see inset of Fig. 5) and $\beta^{\circ}(z=4) \simeq 0.46(2)$.

To summarize, I have studied three different growth models on a substrate of a finite Bethe lattice with different coordination number. A different scaling behavior is seen with respect to the same models on the ordinary lattice or those on the fractal substrates [44]. Two models i.e., the BD and RSOS models, are chosen from the nonequilibrium growth processes in the KPZ universality class, and the third i.e., the RDSR model, is an equilibrium model from EW class. For all considered models, the surface width grows with a power law scaling relation with the logarithm of the time before saturation. The initial growth is characterized by an exponent which depends on the coordination number of the underlying Bethe lattice as well as on the model in question. In the steady state regime, the scaling behavior distinguishes between equilibrium and nonequilibrium models. The average saturated width for the nonequilibrium models has a power law scaling relationship with the logarithm of the lattice size, while for the equilibrium RDSR model, it shows a usual power law behavior with the lattice size (instead of the logarithm of the size).

If we admit that the Bethe lattice, as a substrate of a growth model, reflects the infinite-dimensional limit properties of the models, the present results would then imply that the nonlinear term in the KPZ equation has a relevant contribution at this limit, consequently questioning the existence of a finite upper critical dimension for the KPZ equation.

I would like to thank J. Krug and M. Sahimi for their useful comments, and H. Dashti-Naserabadi for his help with programming. Supports from the Deutsche Forschungsgemeinschaft via SFB/TR 12, and the Humboldt research fellowship are gratefully acknowledged. I also acknowledge partial financial supports by the research council of the University of Tehran and INSF, Iran.
[1] M. Kardar, G. Parisi, and Y-C. Zhang, Phys. Rev. Lett. 56, 889 (1986).

[2] A-L. Barabasi and H. E. Stanley, Fractal Concepts in Surface Growth (Cambridge University. Press, Cambridge, 1995).

[3] J. Krug and H. Spohn, Solids Far From Equilibrium: Growth, Morphology and Defects, edited by C. Godreche (Cambridge University Press, New York, 1991).

[4] D. Forster, D. R. Nelson, and M. J. Stephen, Phys. Rev. A 16, $732(1977)$.
[5] H. van Beijeren, R. Kutner, and H. Spohn, Phys. Rev. Lett. 54, 2026 (1985) [CAS]; H. K. Jansen and B. Schittmann, Z. Phys. B 63, 517 (1986).

[6] D. A. Huse, C. L. Henley, and D. S. Fisher, Phys. Rev. Lett. 55, 2924 (1985); M. Kardar and Y-C. Zhang, Phys. Rev. Lett. 58, 2087 (1987).

[7] J. Z. Imbrie and T. Spencer, J. Stat. Phys. 52, 609 (1988); J. Cook and B. Derrida, Europhys. Lett. 10, 195 (1989); J. Cook and B. Derrida, J. Phys. A 23, 1523 (1990); M. R. Evans and B. Derrida, J. Stat. Phys. 69, 
427 (1992); B. Derrida, H. Spohn, J. Stat. Phys. 51, 817 (1988).

[8] G. Parisi, J. Phys. (Paris) 51, 1595 (1990); M. Mezard, J. Phys. (Paris) 51, 1831 (1990).

[9] E. F. Edwards and D. R. Wilkinson, Proc. R. Soc. London Ser. A 381, 17 (1982).

[10] J. Cook and B. Derrida, J. Phys. A:Math. Gen. 23, 1523 (1990).

[11] J. P. Bouchaud and M. E. Cates, Phys. Rev. E 47, 1455 (1993).

[12] E. Katzav and M. Schwartz, Physica A 309, 69 (2002).

[13] M. Lassig and H. Kinzelbach, Phys. Rev. Lett. 78, 903 (1997).

[14] P. Le Doussal and K. J. Wiese, Phys. Rev. E 72, 035101(R) (2005).

[15] F. Colaiori and M. A. Moore, Phys. Rev. Lett. 86, 3946 (2001).

[16] H. C. Fogedby, Phys. Rev. Lett. 94, 195702 (2005).

[17] E. Perlsman and M. Schwartz, Physica A 234, 523 (1996).

[18] C. Castellano, M. Marsili, and L. Pietronero, Phys. Rev. Lett. 80, 3527 (1998)

[19] T. Ala-Nissila et al., J. Stat. Phys. 72, 207 (1993).

[20] T. Ala-Nissila, Phys. Rev. Lett. 80, 887 (1998); 80, 5459 (1998) (erratum).

[21] J. M. Kim, Phys. Rev. Lett. 80, 888 (1998).

[22] E. Marinari, A. Pagnani, G. Parisi and Z. Rácz, Phys. Rev. E 65, 026136 (2002).

[23] M. Schwartz and E. Perlsman, Phys. Rev. E 85, 050103(R) (2012).

[24] E. Katzav and M. Schwartz, Phys. Rev. E 70, 061608 (2004).

[25] J.M. Kim et al, Phys. Rev. Lett. 62, 2289 (1989).

[26] A. A. Saberi et al, Phys. Rev. E 77, 051607 (2008).

[27] A. A. Saberi and S. Rouhani, , Phys. Rev. E 79, 036102
(2009).

[28] 6. A. A. Saberi, H. Dashti-Naserabadi and S. Rouhani, Phys. Rev. E 82, 020101(R) (2010).

[29] F. Family, J. Phys. A 19, L441 (1986).

[30] H. A. Bethe, Proceedings of the Royal Society of London. Series A, Mathematical and Physical Sciences, 150, No. 871, 552 (1935).

[31] R. J. Baxter, Exactly Solvable Models in Statistical Mechanics (Academic Press, New York, 1982).

[32] M. F. Thorpe, Excitations in Disordered Systems (Plenum Press, New York, 1982).

[33] D. Stauffer and A. Aharony, Introduction to Percolation Theory, 2nd ed. (Taylor \& Francis, London and New York, 1994).

[34] H. Chae, S-H. Yook and Y. Kim, Phys. Rev. E 85, 051118 (2012).

[35] M. Sahimi, Heterogeneous Materials: Linear transport and optical properties, vol 1 (Springer, 2003).

[36] M. Sahimi, AIChE J. 39, 369 (1993).

[37] M. R. Zirnbauer, Phys. Rev. B 34, 63946408 (1986).

[38] J. Krug, J. Phys. A 21, 4637 (1988).

[39] R. M. Bradley and P. N. Strenski, Phys. Rev. B 30, 67886790 (1984).

[40] B. D. Hughes and M. Sahimi, J. Stat. Phys. 29, 781 (1982); Chem. Eng. Sci. 43, 2981 (1988).

[41] D. Cassi, Europhys. Lett. 9, 627 (1989).

[42] M. Alimohammadi, N. Olanj, Physica A 389, 15491554 (2010).

[43] P. Meakin and R. Jullien, J. Phys. (Paris) 48, 1651 (1987); R. Jullien and P. Meakin, Europhys. Lett. 4, 1385 (1987).

[44] S. B. Lee and J. M. Kim, Phys. Rev. E 80, 021101 (2009).

[45] For the sake of simplicity, I use the same symbols for the exponents $\alpha$ and $\beta$, in the logarithmic scaling laws. 